\title{
Odorant binding proteins: a biotechnological tool for odour control
}

\author{
Carla Silva • Teresa Matamá • Nuno G. Azoia • \\ Catarina Mansilha $\cdot$ Margarida Casal $\cdot$ Artur Cavaco-Paulo
}

Received: 4 April 2013 /Revised: 19 August 2013 / Accepted: 21 August 2013

(C) Springer-Verlag Berlin Heidelberg 2013

\begin{abstract}
The application of an odorant binding protein for odour control and fragrance delayed release from a textile surface was first explored in this work. Pig $O B P-1$ gene was cloned and expressed in Escherichia coli, and the purified protein was biochemically characterized. The $\mathrm{IC}_{50}$ values (concentrations of competitor that caused a decay of fluorescence to half-maximal intensity) were determined for four distinct fragrances, namely, citronellol, benzyl benzoate, citronellyl valerate and ethyl valerate. The results showed a strong binding of citronellyl valerate, citronellol and benzyl benzoate to the recombinant protein, while ethyl valerate displayed weaker binding. Cationized cotton substrates were coated with porcine odorant binding protein and tested for their capacity to retain citronellol and to mask the smell of cigarette smoke. The immobilized protein delayed the release of citronellol when compared to the untreated cotton. According to a blind evaluation of 30 assessors, the smell of cigarette smoke, trapped onto the fabrics' surface, was successfully attenuated by porcine odorant binding protein (more than $60 \%$ identified the weakest smell intensity after protein exposure compared to $\beta$-cyclodextrin-treated and untreated
\end{abstract}

Carla Silva and Teresa Matamá contributed equally.

C. Silva · T. Matamá • N. G. Azoia • A. Cavaco-Paulo $(\bowtie)$

IBB - Institute for Biotechnology and Bioengineering,

Centre of Biological Engineering, Universidade do Minho,

Campus de Gualtar, 4710-057 Braga, Portugal

e-mail: artur@deb.uminho.pt

C. Mansilha

Chemical and Toxicology Laboratory, Department of Environmental

Health, Ricardo Jorge Institute, 4000-055 Porto, Portugal

M. Casal

CBMA - Centre of Molecular and Environmental Biology,

University of Minho, Campus Gualtar, 4710-057 Braga, Portugal

T. Matamá

CBMA - Centre of Molecular and Environmental Biology,

Campus Gualtar, 4710-057 Braga, Portugal cotton fabrics). This work demonstrated that porcine odorant binding protein can be an efficient solution to prevent and/or remove unpleasant odours trapped on the large surface of textiles. Its intrinsic properties make odorant binding proteins excellent candidates for controlled release systems which constitute a new application for this class of proteins.

Keywords Odour control · Fragrance · Controlled release . Lipid binding $\cdot$ Lipocalin

\section{Introduction}

The search for new solutions to prevent the development of unpleasant odours and provide long-lasting fragrances is of much interest for both the textiles and cosmetics industries. Textiles have a very large specific surface; thus, they attract, adsorb and store various gaseous compounds from their surroundings. Odour control can be accomplished by the hindrance of unpleasant-smelling substances and/or by the release of fragrances.

The use of odorant binding proteins (OBPs) is a new and exciting strategy for odour control in materials such as textiles with cosmetic, medical and detergent applications. Vertebrate OBPs are small extracellular proteins belonging to the lipocalin superfamily and defined by their property of reversibly binding volatile chemicals, called odorants (Pelosi 1998; Schlehuber and Skerra 2005; Tegoni et al. 2000). They are expressed in the nasal glands of vertebrates and released into the mucus. The first OBP was isolated from bovine nasal mucus; since then they have been identified in a variety of mammalian species besides humans (Bignetti et al. 1985; Pelosi et al. 1982; Pes et al. 1992). Although, after 30 years, their functions are still not fully understood, it is accepted that they actively participate in the transfer of hydrophobic odorous molecules through the aqueous mucus barrier towards the olfactory sensory receptors (Pelosi 1998). It has been 
hypothesized that OBPs play an important role in the events of odour detection by carrying, deactivating and/or selecting odorant molecules. The OBPs share a conserved folding pattern, an eight-stranded $\beta$-barrel flanked by an $\alpha$-helix at the $\mathrm{C}$ terminal end of the polypeptide chain. The $\beta$-barrel creates a central hydrophobic cavity whose role is to bind and transport hydrophobic odorant molecules (Pes et al. 1992).

Porcine odorant binding protein (pOBP), purified from pig nasal mucus, is a monomer of 157 amino acids (with a predicted mass of 17,689 Da) containing one disulphide bridge between cysteine at positions 63 and 155. Its amino acid sequence shares about $42 \%$ of its residues with bovine OBP (Paolini et al. 1998). The structure of pig OBP-I has been already resolved at $2.25 \AA$ resolution (Spinelli et al. 1998) and has revealed a folding very similar to that of the bovine protein, with the exception for the domain swapping, and its monomeric nature (Paolini et al. 1999).

Several applications have been reported for these special proteins. Ramoni et al. (2008) investigated whether these lipocalins have potential to be employed in the design of biosensors for the detection of hazardous compounds. Pietrantonio et al. (2013) studied the binding properties of three OBPs from rat and demonstrated that they were specially tuned towards distinct chemical classes of odorants. Others presented a novel design for a smell nanobiosensor, which integrates mammalian olfactory receptors with an electrical/ electronic transducer (Alfinito et al. 2010). Indeed the progress on the development of electronic noses is associated with the increasing understanding of biochemical, physiological and behavioural aspects of odour sensation where OBPs seem to play a key role (Göpel et al. 1998).

Considering the pertinent challenge for textile industryan efficient solution to prevent and/or remove unpleasant odours trapped on the large surface of textiles - we produced the pOBP and studied its possible textile application. The biochemical characterization of the protein regarding the dissociation constants for several common fragrances was performed. Its adsorption to cationized cotton has also been evaluated as well as its ability to adsorb and attenuate the smell of cigarette smoke on cotton fabrics. For the first time, odorant binding proteins, involved in odour recognition, were explored as a new bio tool to achieve odour control, having textiles as model of study.

\section{Materials and methods}

\section{Reagents}

The ligands, 1-amino anthracene (1-AMA) and fragrances citronellol, benzyl benzoate, citronellyl valerate and ethyl valerate (Table 1), used for protein biochemical characterization, were purchased from Sigma (Madrid, Spain). The fabric used for mask and adsorption experiments was a $100 \%$ bleached cotton ( 33 picks $\times 23$ ends $/ \mathrm{cm}^{2}$ ). Dimethyloctadecyl [3(trimethoxysilyl) propyl] ammonium chloride and all the other chemicals were purchased from Sigma (Madrid, Spain).

Gene cloning, protein expression and purification

The bacterial hosts used for cloning and expression of $O B P-1$ gene from Sus scrofa were the Escherichia coli strain XL1Blue (Agilent Technologies, Santa Clara, CA, USA) and strain BL21 (DE3) (New England BioLabs Inc., Ontario, Canada), respectively. The gene encoding the protein bearing the $\mathrm{F} 88 \mathrm{~W}$ mutation was kindly supplied by Prof. Paolo Pelosi cloned in pET-5b (Wei et al. 2008). Standard techniques were used for all the DNA manipulations. The construct pET-5b carrying pig $O B P-1$ gene bearing the $\mathrm{F} 88 \mathrm{~W}$ mutation was doubledigested with $N d e I$ and $E c o$ RI restriction enzymes (Roche), and the resultant DNA fragment was extracted and purified from agarose gel and cloned into the $N d e \mathrm{I} / E c o$ RI restricted and dephosphorylated pET-28a (Novagene, Merck Millipore, Darmstadt, Germany), resulting in the final pET-28::pOBP construct. The construct pET-28::pOBP was first established in E. coli XL1-Blue strain. Medium-scale purifications of plasmid DNA were made and used to transform the $E$. coli BL21 (DE3) strain. A clone harbouring the construct was grown overnight, at $30{ }^{\circ} \mathrm{C}$ and $200 \mathrm{rpm}$, in $2.5 \mathrm{~L}$ LuriaBroth medium supplemented with ampicillin $\left(100 \mu \mathrm{g} \mathrm{mL}^{-1}\right)$ and $0.2 \% \alpha$-lactose. The cells were harvested by centrifugation and frozen at $-80{ }^{\circ} \mathrm{C}$. The ultrasonic disruption of the bacterial cells was accomplished on ice with a $25.4-\mathrm{mm}$ probe in an Ultrasonic Processor VCX-400 watt (Cole-Parmer Instrument Company, IL, USA). The lysate was centrifuged for $30 \mathrm{~min}$ at $30,000 \mathrm{~g}$ and $4{ }^{\circ} \mathrm{C}$. Imidazole was added to a final concentration of $20 \mathrm{mM}$, and the $\mathrm{pH}$ was adjusted to 7.6. Porcine OBP purification was done by affinity chromatography using HiTrap Chelating HP system (GE Healthcare BioSciences Europe GmbH, Munich, Germany) coupled to a peristaltic pump. In the pOBP-enriched fractions, the elution buffer was changed to phosphate buffer with HiTrap Desalting 20-mL column (GE Healthcare Bio-Sciences Europe GmbH, Munich, Germany). The fractions obtained were monitored by SDS-PAGE with Coomassie staining (Fig. 1).

Fluorescence binding assays

Emission fluorescence of solutions was recorded on a microplate spectrofluorometer with thermoregulation (Synergy Mx, BioTek, Bad Friedrichshall, Germany) at either 25 or $37^{\circ} \mathrm{C}$ with 10 -nm slits for both excitation and emission. The protein was diluted in $50 \mathrm{mM}$ Tris- $\mathrm{HCl}$ buffer, $\mathrm{pH} 7.5$, to a final concentration of $1 \mu \mathrm{M}$, while ligands were added as ethanolic solutions. 
Table 1 Some fragrance physical and chemical properties

\begin{tabular}{|c|c|c|c|c|c|}
\hline Fragrance & Chemical structure & $\begin{array}{l}\mathrm{Mw} \\
\left(\mathrm{gmol}^{-1}\right)\end{array}$ & $\begin{array}{l}\text { Volume } \\
\left(\AA^{3}\right)\end{array}$ & $\begin{array}{l}\log \mathrm{P} \\
(\mathrm{o} / \mathrm{w})\end{array}$ & $\begin{array}{l}\text { Surface } \\
\text { contact } \\
\text { area }\left(\AA^{2}\right)\end{array}$ \\
\hline $\begin{array}{l}\text { Citronellol } \\
\text { (fresh rose) }\end{array}$ & & 156.27 & 182 & 3.91 & 408.7 \\
\hline $\begin{array}{l}\text { Citronellyl valerate } \\
\text { (herbaceous rosy) }\end{array}$ & & 240.38 & 104 & 5.87 & 552.0 \\
\hline $\begin{array}{l}\text { Ethyl valerate } \\
\text { (powerful ethereal apple) }\end{array}$ & & 130.19 & 141 & 1.87 & 330.1 \\
\hline $\begin{array}{l}\text { Benzyl benzoate } \\
\text { (faint balsamic) }\end{array}$ & & 212.24 & 198 & 3.97 & 497.1 \\
\hline
\end{tabular}

To measure the affinity of the fluorescent ligand 1-AMA to the recombinant pOBP, $1 \mu \mathrm{M}$ pOBP in $50 \mathrm{mM}$ Tris- $\mathrm{HCl}$, $\mathrm{pH} 7.5$, was titrated with aliquots of the ligand ethanolic solution to final concentrations of $1-10 \mu \mathrm{M}$ (Paolini et al. 1999; Wei et al. 2008). The data were collected and fitted according to Eq. 1 (Malpeli et al. 1998; Norris and Li 1998). Y is the measured fluorescence ratio, $[\mathrm{AMA}]_{0}$ is the initial concentrations of 1-AMA and $P_{0}$ is the initial concentration of protein; $\mathrm{Kd}$ is the dissociation constant that is calculated by non-linear fitting (OriginPro evaluation demo, OriginLab Corporation, Northampton, MA, USA).

$Y=\frac{[A M A]_{0}+P_{0}+K d-\sqrt{\left([A M A]_{0}+P_{0} K d\right) 2-4 P_{0}[A M A]_{0}}}{2 P_{0}}$

The $\mathrm{IC}_{50}$ values (concentrations of competitor that caused a decay of fluorescence to half-maximal intensity) for the fragrances were measured in competitive binding assays, using $2.5 \mu \mathrm{M}$ 1-AMA as the fluorescent reporter and $1-10 \mu \mathrm{M}$ of citronellol, benzyl benzoate, citronelyl valerate or $1-100 \mu \mathrm{M}$ of ethyl valerate. First-order decay fitting of relative fluorescence enabled the calculation of the $\mathrm{IC}_{50}$.

Capacity of immobilized pOBP to retain fragrances on cotton

The ability of pOBP to mask odours and release fragrances was evaluated using bleached cotton fabrics as the textile support. The fabric samples were previously cationized in order to promote the negatively charged protein surface attachment.

Cotton fabrics cationization

Bleached cotton fabrics were cationized using a cold padbatch technique according to a previously reported method (Hashem et al. 2010). Ten grams per liter of dimethyloctadecyl[3-(trimethoxysilyl)propyl]ammonium chloride was mixed with $4 \mathrm{~g} / \mathrm{L}$ sodium hydroxide and then diluted with water to a final volume of $100 \mathrm{~mL}$. Bleached fabrics were incubated with this solution for $4 \mathrm{~h}$ and then batched in a plastic bag at room temperature overnight. The fabrics were washed with distilled water and finally dried at room temperature conditions. 


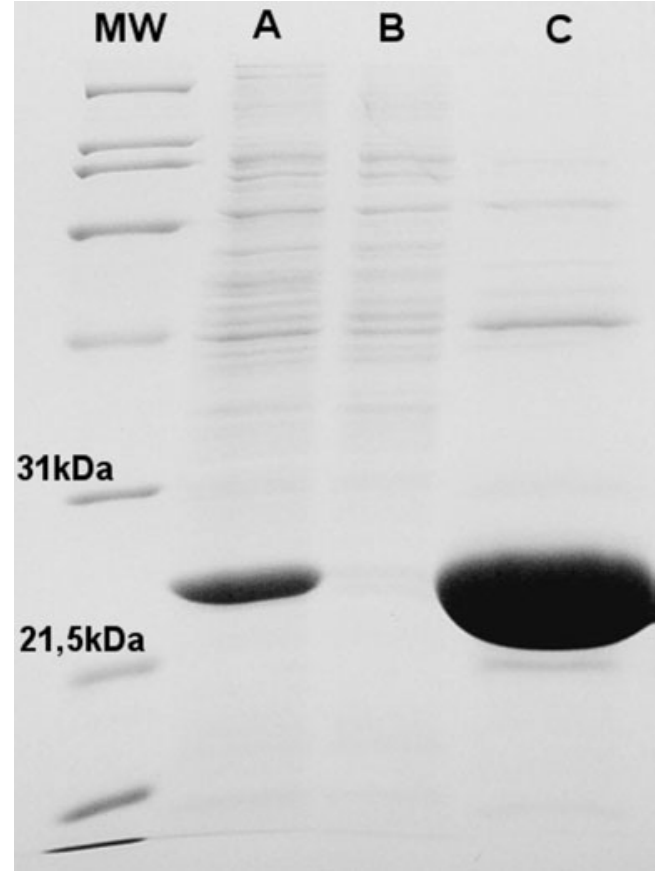

Fig. 1 SDS-PAGE (12\% acrylamide/bisacrylamide) with Coomassie staining of different fractions obtained during pOBP purification. Lane $A$ cell lysate after centrifugation; lane $B$ cell lysate recovered from the affinity column; lane $C$ eluted purified pOBP fraction pool

\section{Adsorption of pOBP to cotton fabrics}

The levels of pOBP adsorption to cotton samples, either cationized or not, were indirectly determined using Bradford's methodology. Fabrics $(5 \times 2.5 \mathrm{~cm})$ were immersed in $5 \mathrm{~mL}$ of either 50 or $100 \mu \mathrm{M}$ pOBP in $50 \mathrm{mM}$ Tris $-\mathrm{HCl}$ buffer, $\mathrm{pH} 7.5$, and incubated at room temperature during
$24 \mathrm{~h}$. Total protein was quantified in the solution, and the percentage of adsorption was calculated regarding the initial protein concentration.

Fragrance release property of functionalized cotton determined by headspace gas chromatography-mass spectrometry (GC-MS)

In order to quantify the concentration of citronellol released from cotton coated with pOBP-citronellol complex and compare it with the amount released from untreated cotton, gas chromatography with a headspace sampler was applied. Cationized cotton samples of $5 \times 2.5 \mathrm{~cm}$ were immersed in $3.4 \mu \mathrm{M}$ pOBP $(\mathrm{pH} 7.5$ ) solution at room temperature, overnight, using appropriate chromatography vials, followed by the addition of $6.8 \mu \mathrm{M}$ of citronellol. Chromatography was carried out on different samples: (a) gas phase in equilibrium with cotton fabric sample immersed in the fragrance pOBP solution, (b) gas phase in equilibrium with a wetted fabric after removing the fragrance solution and (c) gas phase in equilibrium with a fabric sample after drying for $1 \mathrm{~h}$ at $37{ }^{\circ} \mathrm{C}$ (Fig. 2).

Gas chromatography was carried out using a GC-2010 instrument by Shimadzu (Duisburg, Germany) equipped with GC-MS-QP 2010 Plus autosampler and split/splitless injector, ZB-5MS-Guardian polar column $(40 \mathrm{~m} \times 0.25 \mathrm{~mm} \times 0.25 \mu \mathrm{M})$ and a Shimadzu detector. The carrier gas was helium He N60 at a constant flow rate of $1 \mathrm{ml} / \mathrm{min}$. The oven temperature was programmed as follows: isothermal $\left(45^{\circ} \mathrm{C}\right)$ during $5 \mathrm{~min}$ and then increased from 45 to $200{ }^{\circ} \mathrm{C}$ at $7 \%$ min increments. The injector was set at $250^{\circ} \mathrm{C}$. The GC-MS measurements were
Fig. 2 Scheme of the different headspace measurements made by GC-MS using polydimethylsiloxane-SPME fibre assembly, corresponding to three different sample stages: a gas phase in equilibrium with cotton fabric sample immersed in the fragrance pOBP solution, b gas phase in equilibrium with a wetted fabric after removing the fragrance solution, $\mathbf{c}$ gas phase in equilibrium with a fabric sample after drying for $1 \mathrm{~h}$ at $37^{\circ} \mathrm{C}$

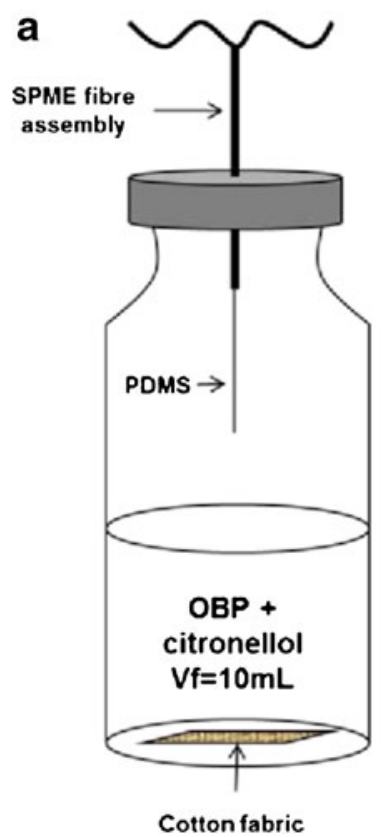

b

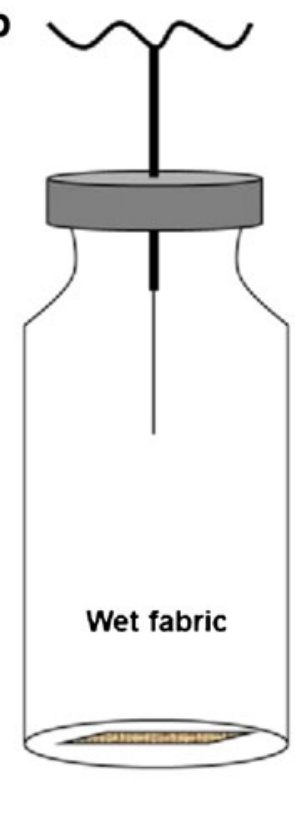

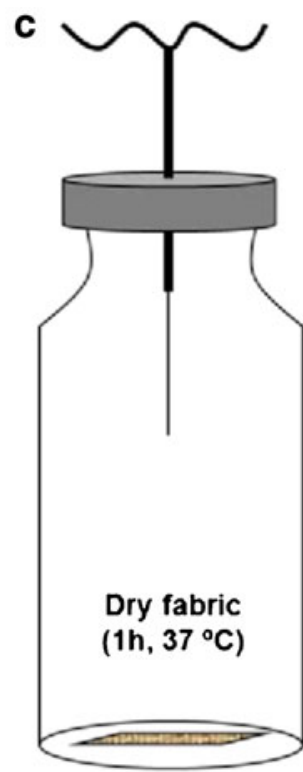


done by pre-incubating the vial containing the cotton sample for $5 \mathrm{~min}$ at $37{ }^{\circ} \mathrm{C}$. The SPME fibre assembly (polydimethylsiloxane-Supelco, SigmaAldrich, Sintra, Portugal ) was then injected $24 \mathrm{~mm}$ in the gas phase inside the vial for 5 min under horizontal agitation $(250 \mathrm{rpm})$. The time for fragrance desorption in the GC injector was set for $10 \mathrm{~min}$. The identification ions for citronellol were 95, 69 and 67, 69 being the quantification ion. The retention time was $15.5 \mathrm{~min}$, and total run time was $27.4 \mathrm{~min}$. A calibration curve was done using five citronellol concentrations applied under the same conditions.

Qualitative assessment of the adsorbed pOBP capacity to mask the smell of cigarette smoke

Cigarette smoke was chosen as model to test the capacity of pOBP to mask bad odours. Three cotton fabrics $\left(4 \mathrm{~cm}^{2}\right)$ were wet with $250 \mu \mathrm{L}$ of distilled water, $260 \mu \mathrm{M} \beta$-cyclodextrin and $260 \mu \mathrm{M}$ pOBP, respectively, and dried at $40{ }^{\circ} \mathrm{C}$. In an Erlenmeyer flask with a two-hole rubber stopper, a cigarette (Belmont, Cigarrera Biggott, Caracas, Venezuela) was fixed at the end of one tube and a syringe was coupled to the other tube. Fifty cubic meters of smoke was aspirated into the Erlenmeyer flask, and the fabrics were exposed to smoke for 45 s. After exposure, the fabrics were kept in separate plastic Petri dishes. The fabrics were then classified according to the relative smoke smell intensity by 32 different individuals (non-smoking and healthy with ages between 20 to 50 years old) in a blind test. A chi-square test of association was performed using Minitab 16 version 16.1.0 (Minitab Inc., State College, PA, USA). The null hypothesis was that there was no association between the type of treatment of cotton samples and the intensity of cigarette smell. When $P$-values were below 0.05 , the differences were considered significant.

\section{Surface contact area}

The surface contact area (Conte et al. 1999) was calculated using Gromacs package (Hess et al. 2008). The starting configurations of the complexes between the fragrances and the protein were obtained using AutoDock Vina (Trott and Olson 2010), and each complex was allowed to relax for $30 \mathrm{~ns}$.

\section{Results}

Biochemical characterization of recombinant pig OBP-1

The expression of pig $O B P-1$ gene in $\mathrm{pET}-28$ vector produced a recombinant protein with 178 amino acids and an estimated molecular weight and isoelectric point of 20,056 Da and 4.7, respectively. The extra 6-histidine tag at the N-terminal allowed the easy purification of pOBP through nickelchelating affinity chromatography. The pOBP production yielded $290 \mathrm{mg}$ of total protein at $96 \%$ relative purity (according to gel analysis using Image J 1.46r software (Wayne Rasband, National Institutes of Health, Bethesda, MD, USA) per liter of bacterial culture.

The dissociation constants for 1-AMA and $\mathrm{IC}_{50}$ values for four different fragrances, common in textile applications, were determined at two different temperatures - ambient temperature $\left(25^{\circ} \mathrm{C}\right)$ and body temperature $\left(37^{\circ} \mathrm{C}\right)$. The odorant molecules tested in this work, all used in the industry, have been chosen with different chemical structures and natures in order to find different interaction strengths between pOBP and odour molecules. They are either aromatic (benzyl benzoate) or aliphatic (citronellol, citronellyl valerate and ethyl valerate), and they possess several types of functional groups (alcohols, aldehydes, esters) and are of different size and hydrophobic character (Table 1).

Figure 3 shows the binding curves of 1-AMA to the recombinant pOBP obtained at 25 and $37^{\circ} \mathrm{C}$. The dissociation constants were obtained by mathematical fitting of data according to Eq. 1. The values for the dissociation constants were $0.16 \pm 0.04$ and $0.09 \pm 0.02 \mu \mathrm{M}$ at 25 and $37^{\circ} \mathrm{C}$, respectively. A maximum saturation of pOBP was observed at around $2 \mu \mathrm{M}$ of 1-AMA, corresponding to an apparent stoichiometry of two molecules of 1-AMA to one molecule of pOBP. The dissociation constants obtained for 1-AMA, using this recombinant $\mathrm{pOBP}$, show that this reporter binds strongly to this protein and that by raising the temperature its affinity for pOBP increases.

The $\mathrm{IC}_{50}$ values for the different odorant molecules were determined by competition assays (Fig. 4 and Table 2). It is possible to distinguish three odorant groups regarding their

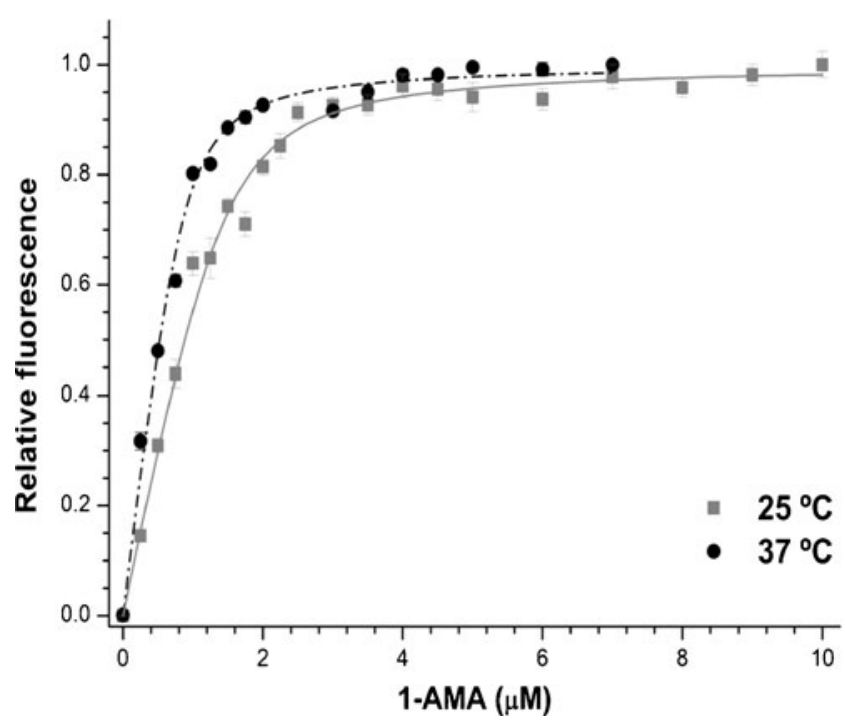

Fig. 3 Binding curves of recombinant $\mathrm{pOBP}$ obtained by measuring the fluorescence of $1 \mu \mathrm{M}$ pOBP in $50 \mathrm{mM}$ Tris- $\mathrm{HCl}, \mathrm{pH} 7.5$, at equilibrium with several concentrations of 1-aminoanthracene. The dissociation constants were obtained at two temperatures, $25{ }^{\circ} \mathrm{C}(\mathrm{Kd}=0.16 \pm 0.04 \mu \mathrm{M})$ and $37^{\circ} \mathrm{C}(\mathrm{Kd}=0.09 \pm 0.02 \mu \mathrm{M})$, by mathematical fitting of data 

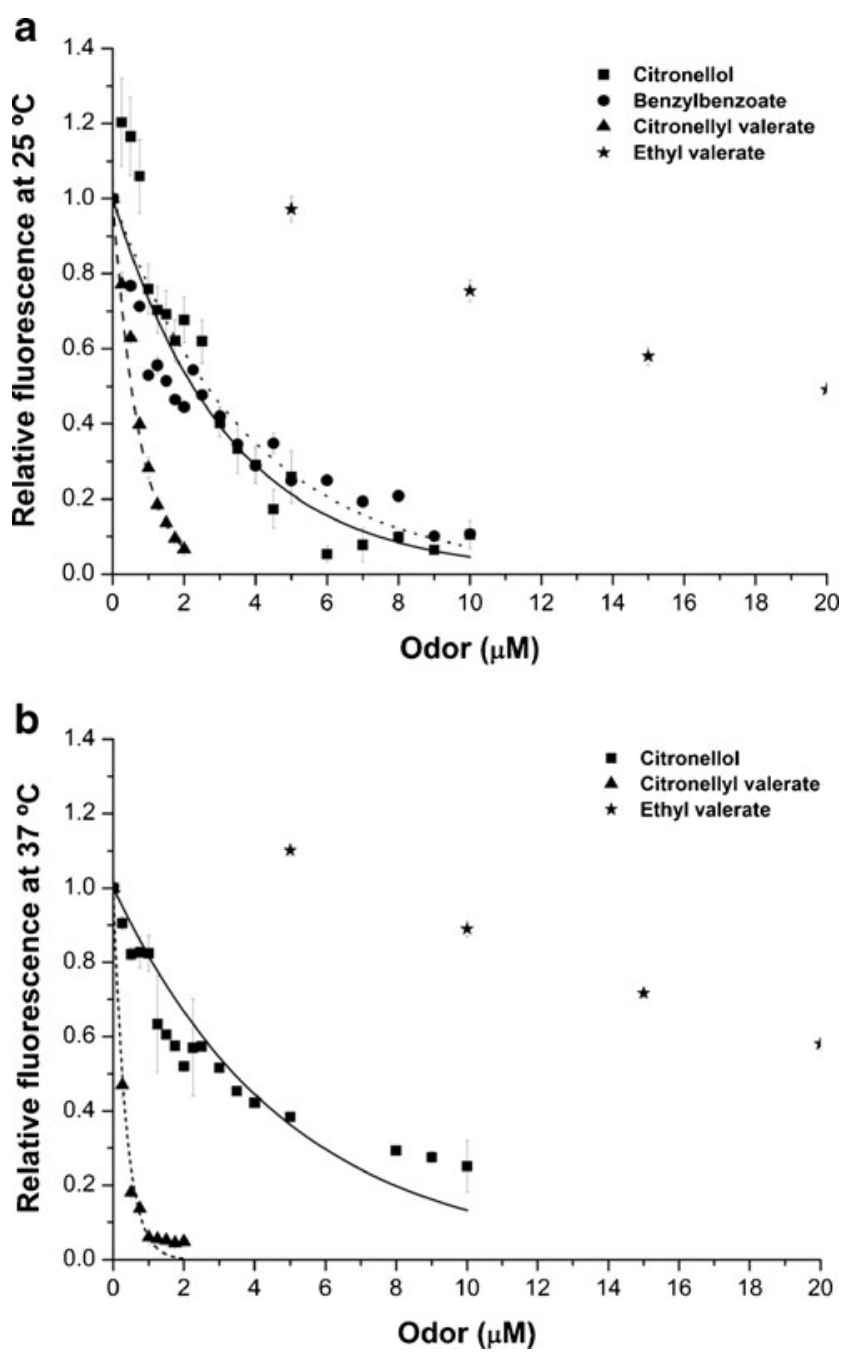

Fig. 4 Competitive binding to pOBP of several fragrances (citronellol, citronelyll valerate, benzyl benzoate, and ethyl valerate) at $25^{\circ} \mathrm{C}$ (a) and $37{ }^{\circ} \mathrm{C}(\mathbf{b})$. The protein and the fluorescent probe 1-aminoanthracene concentrations used were 1 and $2.5 \mu \mathrm{M}$, respectively, while competitors were added as $1-10 \mu \mathrm{M}$ ethanolic solutions

binding strength for pOBP. Citronellyl valerate is the molecule with the greatest ability to displace 1-AMA from the binding pocket of pOBP (highly potent: $\mathrm{IC}_{50} \leq 1 \mu \mathrm{M}$ ), followed by citronellol and benzyl benzoate, both with similar affinities for pOBP (moderately potent: $\mathrm{IC}_{50} \leq 100 \mu \mathrm{M}$ ). From the tested

Table $2 \mathrm{IC}_{50}$ values for the studied fragrances determined by competitive binding with 1-AMA-pOBP

\begin{tabular}{lcc}
\hline Fragrance & $\mathrm{IC}_{50}(\mu \mathrm{M})$ & \\
\cline { 2 - 3 } & $25{ }^{\circ} \mathrm{C}$ & $37{ }^{\circ} \mathrm{C}$ \\
\hline Citronellol & $2.2 \pm 0.1$ & $3.4 \pm 0.1$ \\
Citronellyl valerate & $0.52 \pm 0.01$ & $0.24 \pm 0.01$ \\
Ethyl valerate & $38 \pm 3$ & $47 \pm 2$ \\
Benzylbenzoate & $2.6 \pm 0.1$ & - \\
\hline
\end{tabular}

fragrances, ethyl valerate has the lowest affinity for pOBP, although according to the accepted classification, it was considered moderately potent. This behaviour is similar at both temperatures $\left(25\right.$ and $\left.37^{\circ} \mathrm{C}\right)$. At $37^{\circ} \mathrm{C}$, there was a significant increase in the affinity of citronellyl valerate for pOBP: twofold reduction in $\mathrm{IC}_{50}$. In contrast, citronellol and ethyl valerate displaced 1-AMA from pOBP less efficiently with the temperature rise as seen by the slight increases in $\mathrm{IC}_{50}$ values. Temperature dependence was different among the tested fragrances, but only for potent ligands, namely, 1-AMA and citronellyl valerate; this parameter particularly affected the binding strength. At $37^{\circ} \mathrm{C}$, due to less stable fragrance concentrations during the incubation, the curve fitting was slightly affected and the measurement of benzyl benzoate binding was precluded.

There is a very significant negative correlation between the logarithm of $\mathrm{IC}_{50}$ and hydrophobicity measured by the octanol-water partition coefficient, $\log P$ values, for the tested compounds (Tables 1 and 2): the Pearson correlation coefficient is -0.961 and the $P$-value is 0.009 . Other properties, such molecular size, are not correlated with $\mathrm{Kd}$ as found by others (Pevsner et al. 1990).

Odour control properties of immobilized pOBP

The ability of pOBP to release fragrance/mask bad odours from a textile surface was tested in this work using a previously cationized cotton fabric as substrate. At neutral $\mathrm{pH}$, the

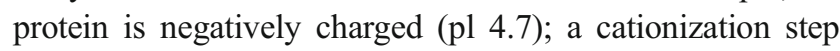
confers a positive charge to the surface of cotton, therefore increasing protein adsorption through electrostatic interactions. This was confirmed experimentally by measuring protein adsorption to the textile as the difference in the amount present in solution after $24 \mathrm{~h}$ in contact with cotton fabric samples (Fig. 5). For both tested concentrations, more than $75 \%$ of initial protein was adsorbed to cationized samples after $24 \mathrm{~h}$. For regular cotton samples, the protein did not get adsorbed under the conditions used.

To characterize the performance of immobilized pOBP in the release of fragrances from cotton, the molar proportion of pOBP-citronellol was set at 1:2, and the amount of protein was dictated by the chosen amount of citronellol to avoid the

Table 3 Citronellol concentration determined by headspace gas chromatography-mass spectrometry released from control $(6.8 \mu \mathrm{M}$ citronellol $)$ and cotton functionalized with pOBP-citronellol (1:2 molar proportion)

\begin{tabular}{llll}
\hline Cotton fabric & \multicolumn{2}{l}{ Citronellol $(\mu \mathrm{M})$} & \\
\cline { 2 - 4 } & In solution & Wet & Dry $\left(1 \mathrm{~h}\right.$ at $\left.37^{\circ} \mathrm{C}\right)$ \\
\hline Control fabric & 5.32 & 4.80 & 3.08 \\
Fabric + pOBP & 2.86 & 3.09 & 2.33 \\
\hline
\end{tabular}




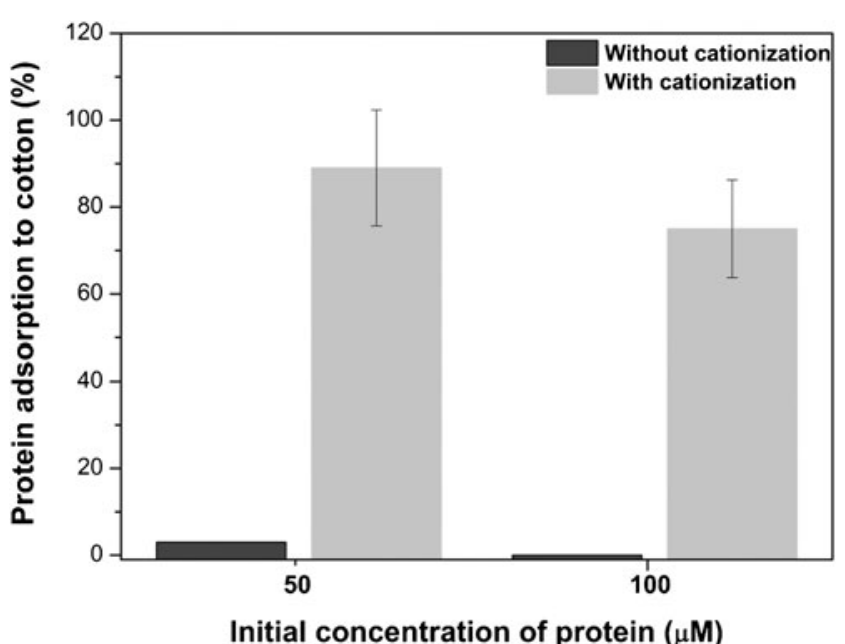

Fig. 5 Protein adsorption to non-cationized and cationized cotton samples after $24 \mathrm{~h}$ of incubation with two different initial concentrations of pOBP

MS detector saturation. Three sample stages were measured independently (Fig. 2): (a) gas phase in equilibrium with cotton fabric sample immersed in the fragrance pOBP solution, (b) gas phase in equilibrium with a wetted fabric after removing the fragrance solution and (c) gas phase in equilibrium with a fabric sample after drying for $1 \mathrm{~h}$ at $37^{\circ} \mathrm{C}$. From the results obtained in a preliminary study (Table 3 ), the quantities of fragrance released either from solution or from the fabric coated with pOBP directly exposed to air are always below the amount released from the untreated cotton and more constant. Considering the initial values, corresponding to the stage where the fabric is still immersed in the solution containing citronellol, the release of fragrance from the wet control fabric was $90 \%$ of the amount released at the first stage, while the amount released from the pOBP-treated sample was $108 \%$. On the sample dried for $1 \mathrm{~h}$ before measurement, the amount released from the control was just $58 \%$, while for the pOBP-treated sample it was $81 \%$. This preliminary assay shows an interesting delaying property of $\mathrm{pOBP}$ in the fragrance released from fabric samples.

To establish if pOBP would be efficient in eliminating or reducing bad odours from cloth, a small-scale qualitative assay was performed. After impregnation of cotton fabric samples with pOBP, $\beta$-cyclodextrin (a known anti-odour textile finish) and water, they were exposed to cigarette smoke for $45 \mathrm{~s}$. A panel of 32 people was asked to order ascendently the three samples according to the perceived cigarette smell intensity. Two assessors were not able to distinguish any different smell intensities among the three samples; 14 assessors differentiated all the samples and ranked them in three levels of intensity; 16 were able only to distinguish one sample intensity from the others, either weaker or stronger than the other two (Fig. 6). From the pool of assessors that distinguished three different levels of intensity (Fig. 6, light grey slice), $65 \%$ of them evaluated the sample with pOBP as bearing the weakest cigarette smell, while only $20 \%$ classified it as the strongest (Fig. 7a). The differences among the smell intensities profiles are significant $(P<0.05)$. From the pool of individuals that classified at least one sample as different from the others (Fig. 5, medium grey), $80 \%$ of them classified the sample containing pOBP as the weakest of all (Fig. 7b).

Samples impregnated with cyclodextrin or water were predominantly classified as possessing stronger cigarette smell intensity. The differences among the smell intensity profiles are very significant $(P<0.01)$, revealing a strong association between cigarette smell intensity and sample treatments.

\section{Discussion}

Studies of the binding specificity of OBPs towards several common odorants, which were performed mostly with bovine OBP and porcine OBP-I (pOBP), have revealed that OBPs can bind a broad spectrum of hydrophobic molecules of medium size (Stepanenko et al. 2012). The industrial application of these proteins in odour control or as general microcapsules is very appealing due to their broad specificity and their thermo-stability (up to $60^{\circ} \mathrm{C}$ or up to $65^{\circ} \mathrm{C}$ if in complex with a ligand) (Paolini et al. 1999). The two temperatures studied were chosen to understand and anticipate how temperature may influence fragrance release from pOBP, for instance, when functionalized textiles, coated with pOBP, at room temperature come in contact with the human body. Paolini et al. (1998) obtained a dissociation constant of $1.3 \mu \mathrm{M}$ for 1AMA binding to native porcine OBP-I at $20^{\circ} \mathrm{C}$, and the value remained almost unchanged up to $65^{\circ} \mathrm{C}$. Some authors demonstrated that post-translational modifications of OBPs can further increase the micro-diversity of ligand affinities (Le Danvic et al. 2009; Nagnan-Le Meillour et al. 2009).

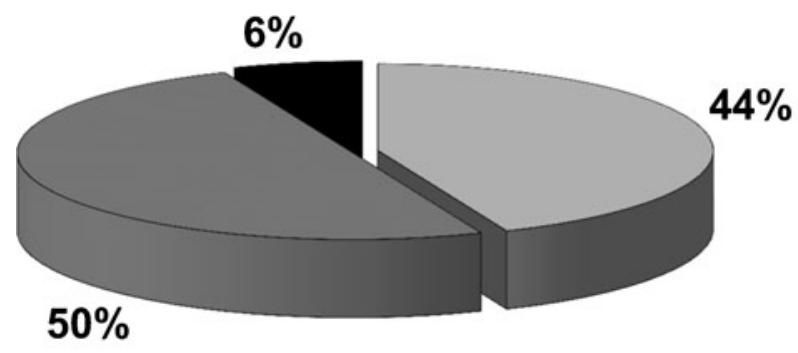

口Individuals that found 3 samples all different

口Individuals that found 1 sample different from the others

- Individuals that did not found any diferences between samples

Fig. 6 Sensitivity profile of 32 different assessors (non-smoking, ages 20-50 years old) to the smell intensity transduced in their ability to distinguish the different samples 
Porcine OBP can be post-translationally modified, resulting in a set of $\mathrm{pOBP}$ isoforms with different binding properties (Brimau et al. 2010). Since the host $E$. coli has a very limited ability to perform enzymatic post-translational modifications of heterologous proteins allied to the extra amino acids coded by the expression vector present at the N-terminus of our recombinant protein and like the mutation $\mathrm{F} 88 \mathrm{~W}$, it would be expected that our pOBP would differ from the native form. The pOBP produced in this work has a higher affinity for 1AMA than the reported affinities for pOBP and, as it happened to citronellyl valerate, raising the temperature increases the binding strength to 1-AMA. The fact that the affinity to a particular fragrance increases just by raising from ambient to human body temperature could be a very interesting property for a textile application of OBPs. In one way, it could mean a better capture of body odours during apparel wearing. Moreover, the application of OBPs to textiles could delay the release of a fragrance, allowing for longer-lasting scents during apparel wearing when compared with the textiles without OBP treatment.

According to the literature, heterocyclic derivatives are among the ligands with the highest affinity for OBPs; thiazoles, terpenoids and their derivatives as well as mediumsized aliphatic alcohols and aldehydes also have good affinity for OBP (Stepanenko et al. 2012). Spherically shaped terpenoids, such as camphor and its analogues, and polar compounds, such as the short-chain fatty acids, exhibit poor affinity to OBPs. Vincent et al. (2000) have studied the pOBP binding to molecules very different in chemical nature and structure, with different functional groups. According to their results, native pOBP bonded tp the selected odorant molecules with good affinity at the micromolar range, the same range found for the recombinant pOBP heterologously produced and purified by us. They also observed that fragrances composed of a single hydrocarbon chain or having a substantially aliphatic structure appeared to be better ligands when compared to short alkyl chain-substituted or non-substituted aromatic molecules (Vincent et al. 2000). Our results also revealed a high affinity for the single aliphatic chain molecule (citronellyl valerate) compared with a shorter aliphatic molecule (citronellol) and a non-substituted aromatic molecule (benzyl benzoate). A correlation between fragrance surface contact area and their affinity to the protein can be established. Higher values of surface contact area correspond to a higher affinity of the fragrance to the pOBP (Table 1). This profile was observed for both tested temperatures, 25 and $37{ }^{\circ} \mathrm{C}$. From the results attained, it is possible to establish a relation between hydrophobic character and the affinity of the several tested fragrances for pOBP. As hydrophobicity increases, the affinity of these molecules for the odorant binding protein also increases. However, the degree of affinity depends also on many other factors, like the molecular structure and conformation inside the protein cavity (Dal Monte et al. 1993). Within the four odorants tested, we have found significant differences between odorant molecule affinities. Therefore, different releasing profiles according to a particular chosen fragrance should be attained for the immobilized pOBP. The fact that OBP is able to interact with a broad range of markedly different structures and does not display a uniquely high affinity for any single chemical class (Pevsner et al. 1990) is determinant for a successful application of these proteins for odour control in textiles and/or fragrance release.

The fragrance-finished textiles, relatively new in the market, are gaining increasing attention and importance. Since fragrance materials have poor water solubility and they usually exist in a liquid state, the perfuming process constitutes a
Fig. 7 Sample blind classification according to the most sensitive assessors that considered the three samples different (a) and the classification according to the assessors that classified at least one sample to be different from the others (b). A chi-square test showed that there was some association between cigarette smell intensity and cotton treatment when three distinct intensities were differentiated ( $\chi^{2}$ 9.857; d.f. 4; $P$-value $=0.043)$ and a strong association when two smell intensities were distinguished $\left(\chi^{2}\right.$ 10.685; d.f. $2 ; P$-value $=0.005$ ) a
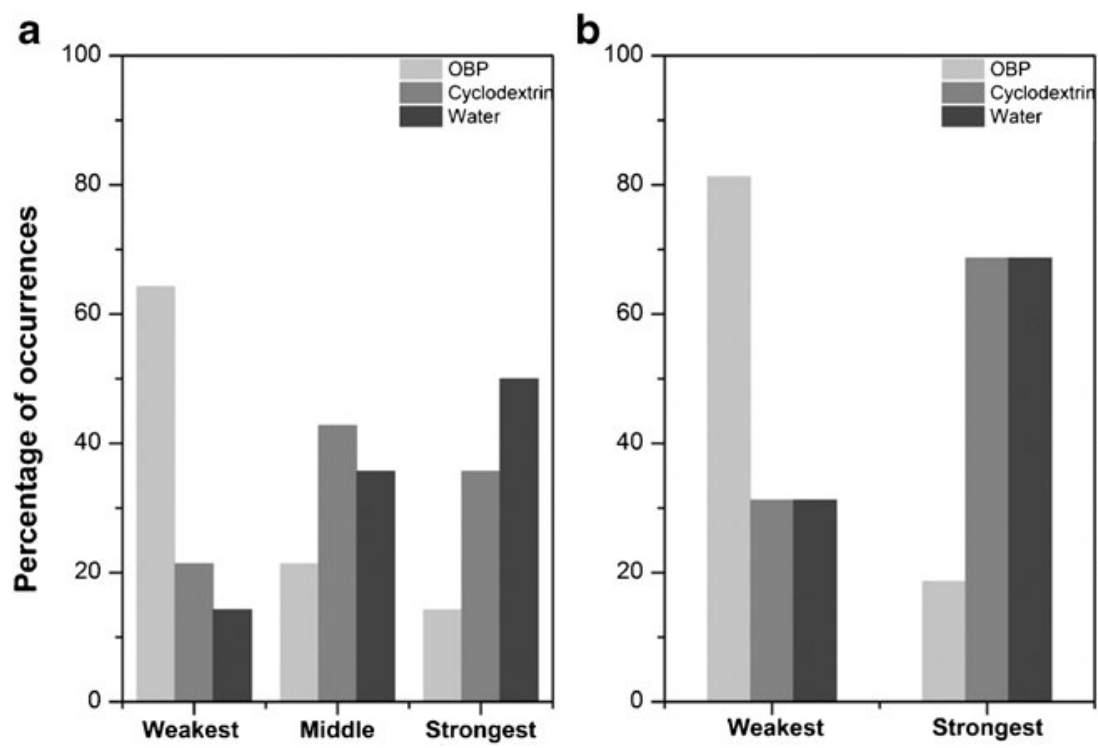

Cigarette smell intensity 
major problem for the textile industry. In addition, the amount of fragrance in the product rapidly decreases during storage due to their volatility and poor stability ( $\mathrm{Hu} 2011)$. These challenges for the textile industry led us to develop a novel methodology involving the electrostatic immobilization of porcine OBP on cotton. A cotton surface, previously cationized, was efficiently coated with pOBP, and due to its intrinsic properties, this protein was able to delay the release of citronellol at $37^{\circ} \mathrm{C}$ under mechanical agitation as measured by headspace GC-MS (Table 3).

A sensorial evaluation of cigarette smell intensity was performed by a group of 32 subjects. The panel evaluation results revealed that porcine OBP masked the cigarette smell from exposed cotton samples more efficiently than the untreated control and cotton samples treated with a popular auxiliary textile substance- $\beta$-cyclodextrin (Fig. 7) (Martel et al. 2002; Szejtli 2003). The capacity to capture a smell composed of such diversity of molecules (like the unpleasant smell of cigarette smoke) is very promising.

Porcine OBP was shown to be more efficient on removing bad odours than cyclodextrins, opening new possibilities for their application where cyclodextrins are used: self-cleaning (by capturing lipid molecules), prevention of malodour development, prolonged release of fragrances as well as other molecules such as antimicrobial agents and insect repellents. As a protein, pOBP can be immobilized covalently, for instance, using glutaraldehyde, improving its washing fastness, and it can be used as the protein basis for nanocarrier production and complex surfactants, opening numerous possibilities not only in the textile industry but also in pharmacy, food and cosmetic industries among others.

Acknowledgments This work was co-funded by the European Social Fund through the management authority POPH and FCT. The authors Carla Silva and Teresa Matamá would like to acknowledge their postdoctoral fellowships: SFRH/BPD/46515/2008 and SFRH/BPD/47555/ 2008, respectively.

\section{References}

Alfinito E, Millithaler JF, Pennetta C, Reggiani L (2010) A single protein based nanobiosensor for odorant recognition. Microelectr J 41(11): 718-722. doi:10.1016/j.mejo.2010.07.006

Bignetti E, Cavaggioni A, Pelosi P, Persaud KC, Sorbi RT, Tirindelli R (1985) Purification and characterisation of an odorant-binding protein from cow nasal tissue. Eur J Biochem 149(2):227-231. doi:10. 1111/j.1432-1033.1985.tb08916.x

Brimau F, Cornard J-P, Le Danvic C, Lagant P, Vergoten G, Grebert D, Pajot E, Nagnan-Le Meillour P (2010) Binding specificity of recombinant odorant-binding protein isoforms is driven by phosphorylation. J Chem Ecol 36(8):801-813. doi:10.1007/s10886-010-9820-4

Conte LL, Chothia C, Janin J (1999) The atomic structure of proteinprotein recognition sites. J Mol Biol 285(5):2177-2198. doi:10. 1006/jmbi.1998.2439
Dal Monte M, Centini M, Anselmi C, Pelosi P (1993) Binding of selected odorants to bovine and porcine odorant-binding proteins. Chem Senses 18(6):713-721. doi:10.1093/chemse/18.6.713

Di Pietrantonio F, Cannatà D, Benetti M, Verona E, Varriale A, Staiano M, D'Auria S (2013) Detection of odorant molecules via surface acoustic wave biosensor array based on odorantbinding proteins. Biosens Bioelectron 41:328-334. doi:10. 1016/j.bios.2012.08.046

Göpel W, Ziegler C, Breer H, Schild D, Apfelbach R, Joerges J, Malaka R (1998) Bioelectronic noses: a status report part I. Biosens Bioelectron 13(3-4):479-493. doi:10.1016/S09565663(97)00092-4

Hashem M, El-Bisi M, Sharaf S, Refaie R (2010) Pre-cationization of cotton fabrics: an effective alternative tool for activation of hydrogen peroxide bleaching process. Carbohyd Polym 79(3):533-540. doi: 10.1016/j.carbpol.2009.08.038

Hess B, Kutzner C, van der Spoel D, Lindahl E (2008) GROMACS 4: algorithms for highly efficient, load-balanced, and scalable molecular simulation. J Chem Theory Comput 4(3):435-447. doi:10.1021/ ct700301q

Hu J (2011) Adaptive and functional polymers, textiles and their applications. Imperial College Press, London

Le Danvic C, Guiraudie-Capraz G, Abderrahmani D, Zanetta J-P, Nagnan-Le Meillour P (2009) Natural ligands of porcine olfactory binding proteins. J Chem Ecol 35(7):741-751. doi:10.1007/s10886009-9645-1

Malpeli G, Folli C, Cavazzini D, Sartori G, Berni R (1998) Purification and fluorescent titration of cellular retinol-binding protein. In: Redfern C (ed) Retinoid protocols. Methods in molecular biology. Human, Totowa, pp 111-122

Martel B, Morcellet M, Ruffin D, Vinet F, Weltrowski L (2002) Capture and controlled release of fragrances by $\mathrm{CD}$ finished textiles. J Inc Phenom Macro 44(1-4):439-442. doi:10.1023/ a: 1023028105012

Nagnan-Le Meillour P, Le Danvic C, Brimau F, Chemineau P, Michalski J-C (2009) Phosphorylation of native porcine olfactory binding proteins. J Chem Ecol 35(7):752-760. doi:10.1007/s10886-0099663-z

Norris AW, Li E (1998) Fluorometric titration of the CRABPs. In: Redfern C (ed) Retinoid protocols. Methods in molecular biology. Humana, Totowa, pp 123-139

Paolini S, Scaloni A, Amoresano A, Marchese S, Napolitano E, Pelosi P (1998) Amino acid sequence, post-translational modifications, binding and labelling of porcine odorant-binding protein. Chem Senses 23(6):689-698. doi:10.1093/chemse/23.6.689

Paolini S, Tanfani F, Fini C, Bertoli E, Pelosi P (1999) Porcine odorantbinding protein: structural stability and ligand affinities measured by Fourier-transform infrared spectroscopy and fluorescence spectroscopy. BBA-Protein Struct M 1431(1):179-188. doi:10.1016/S01674838(99)00037-0

Pelosi P (1998) Odorant-binding proteins: structural aspects. Ann NY Acad Sci 855(1):281-293. doi:10.1111/j.1749-6632.1998. tb10584.x

Pelosi P, Baldaccini NE, Pisanelli AM (1982) Identification of a specific olfactory receptor for 2-isobutyl-3-methoxypyrazine. Biochem J 201(1):245-248

Pes D, Dal Monte M, Ganni M, Pelosi P (1992) Isolation of two odorantbinding proteins from mouse nasal tissue. Comp Biochem Phys B 103(4):1011-1017. doi:10.1016/0305-0491(92)90231-F

Pevsner J, Hou V, Snowman AM, Snyder SH (1990) Odorant-binding protein. Characterization of ligand binding. J Biol Chem 265(11): $6118-6125$

Ramoni R, Staiano M, Bellucci S, Grycznyski I, Grycznyski Z, Crescenzo R, Iozzino L, Bharill S, Conti V, Grolli S, D'Auria S (2008) Carbon nanotube-based biosensors. J Phys-Condens Mat 20(47):474201. doi:10.1088/0953-8984/20/47/474201 
Schlehuber S, Skerra A (2005) Lipocalins in drug discovery: from natural ligand-binding proteins to 'anticalins'. Drug Discov Today 10(1): 23-33. doi:10.1016/S1359-6446(04)03294-5

Spinelli S, Ramoni R, Grolli S, Bonicel J, Cambillau C, Tegoni M (1998) The structure of the monomeric porcine odorant binding protein sheds light on the domain swapping mechanism. Biochemistry 37(22):7913-7918. doi:10.1021/bi980179e

Stepanenko O, Fonin A, Stepanenko O, Kuznetsova I, Turoverov K (2012) Ligand-binding proteins: structure, stability and practical application. In: Faraggi E (ed) Protein structure. InTech, Rijeka, pp 265-290

Szejtli J (2003) Cyclodextrins in the textile industry. Starch - Stärke 55(5): 191-196. doi:10.1002/star.200390050

Tegoni M, Pelosi P, Vincent F, Spinelli S, Campanacci V, Grolli S, Ramoni R, Cambillau C (2000) Mammalian odorant binding proteins. BBA-Protein Struct M 1482(1-2):229-240. doi:10.1016/ S0167-4838(00)00167-9

Trott O, Olson AJ (2010) AutoDock vina: improving the speed and accuracy of docking with a new scoring function, efficient optimization, and multithreading. J Comput Chem 31(2):455-461. doi:10. $1002 /$ jcc. 21334

Vincent F, Spinelli S, Ramoni R, Grolli S, Pelosi P, Cambillau C, Tegoni M (2000) Complexes of porcine odorant binding protein with odorant molecules belonging to different chemical classes. J Mol Biol 300(1):127-139. doi:10.1006/jmbi.2000.3820

Wei Y, Brandazza A, Pelosi P (2008) Binding of polycyclic aromatic hydrocarbons to mutants of odorant-binding protein: a first step towards biosensors for environmental monitoring. BBAProteins Proteom 1784(4):666-671. doi:10.1016/j.bbapap. 2008.01.012 